\title{
Bilateral eyelid swelling: Floppy eyelid syndrome with obstructive sleep apnea
}

\author{
Mika Yamanaka-Takaichi', Hidenori Mito², Nao Kusutani' ${ }^{1}$, Daisuke Tsuruta1

\begin{abstract}
${ }^{1}$ Department of Dermatology, Osaka City University Graduate School of Medicine, Osaka, Japan, ${ }^{2} I d e$ Eye Hospital, Yamagata, Japan
\end{abstract}

Corresponding author: Dr. Mika Yamanaka-Takaichi, E-mail: mikay0115@gmail.com

Sir,

Floppy eyelid syndrome (FES) is an eye disorder associated with obstructive sleep apnea syndrome (OSAS). First described by Culbertson and Ostler in 1981, FES is characterized by "floppy" and redundant upper eyelids with marked papillary conjunctivitis, and typically occurs in obese middle-aged and older men [1].

A 49-year-old man presented with bilateral upper eyelid swelling for several years. He was referred from an ophthalmologist after treatment with eye drops, topical corticosteroids, and steroid injection resulted in no improvement. The patient showed erythema, lichenification, and edema on both sides of the upper eyelids, causing eye-opening difficulty (Fig. la). The clinical differential diagnoses included thyroidassociated ophthalmopathy or dermatomyositis, but blood testing revealed normal levels of muscle-related enzymes (CK, AST, ALT, and LDH) and pituitary/ thyroid hormones, and negative results for antinuclear antibody and anti-aminoacyl-tRNA synthetase antibody. MRI showed hyperplasia of adipose tissues in bilateral eyelids and eye sockets. The patient suffered pollinosis in spring, leading to chronic sinusitis.

The patient's eyelids easily everted with minimal lateral traction, he was obese (body mass index: 25.2), and he suffered from snoring, daytime sleepiness, and fatigue-supporting a diagnosis of FES. A sleep physician provided the patient with an overnight respiratory portable polygraph, which revealed mild OSAS. Due to severe impairment in nocturnal minimum saturation data, the patient began continuous positive air pressure (CPAP) therapy. His eye symptom did not improve after

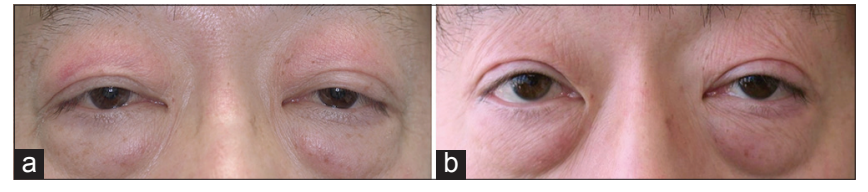

Figure 1: (a) Photo taken before surgery, showing erythema, lichenification, and edema on both sides of the upper eyelids. (b) Photo taken six months after surgery.

6 months, and he therefore underwent corrective eyelid surgery. After 12 months of post-surgery follow-up, the patient shows no apparent recurrence (Fig. lb).

Several eye diseases have been described in association with OSAS, including optic neuropathy, glaucoma, nonarteritic anterior ischemic optic neuropathy (NAION), papilledema secondary to raised intracranial pressure (ICP), and FES [2]. Although FES pathogenesis is unclear, a number of studies clearly indicate a close relationship between FES and OSAS [2-5]. It is suspected that the affected side corresponds to habitual sleeping posture, with both sides being affected if the patient alternates sleeping sides or sleeps face down [4]. Chronic eyelid rubbing and stretching may lead to the observed eyelid changes, and such changes could accelerate once the eyelid begins to spontaneously evert during sleep [2]

Treatments for FES have not been established. Previous studies report that FES might be treated by using a lid shield at night, lid taping, nocturnal lubrication, topical steroids, lid scrubbing, and punctal plugs. Treatment with CPAP corrects apnea/hypopnea events and can reportedly improve daytime sleepiness and FES symptoms [4]. However, in our case, CPAP therapy did not yield substantial improvement of the eye symptoms. Recently, various surgical techniques have been reported

\footnotetext{
How to cite this article: Yamanaka-Takaichi M, Mito H, Kusutani N, Tsuruta D. Bilateral eyelid swelling: Floppy eyelid syndrome with obstructive sleep apnea. Our Dermatol Online. 2019;10(2):206-207.

Submission: $30.08 .2018 ; \quad$ Acceptance: 29.10 .2018

DOI: 10.7241 /ourd.20192.28
} 
for FES [5]. Since FES patients may visit dermatological clinic, not only ophthalmologists but also dermatologists should be aware of these ocular and periocular symptoms that can be associated with sleep apnea.

\section{Consent}

The examination of the patient was conducted according to the Declaration of Helsinki principles.

\section{REFERENCES}

1. Culbertson WW, Ostler HB. The floppy eyelid syndrome. Am J Ophthalmol. 1981;92:568-75.
2. McNab AA. The eye and sleep apnea. Sleep Med Rev. 2007;11:269-76.

3. McNab AA. The eye and sleep. Clin Exp Ophthalmol. 2005;33:117-25.

4. Porta R, Comini L, Barbano L, Bianchi L, Vitacca M. A case of obstructive sleep apnea syndrome associated with floppy eyelid syndrome: positive effect of CPAP therapy. Monaldi Arch Chest Dis. 2017;87:55-6.

5. Lee CC, Lai HT, Kuo YR, Lee SS, Lai CS. Floppy Eyelid Syndrome: An Unfamiliar Entity for Plastic Surgeons. Ann Plast Surg. 2018;80:40- 7.

Copyright by Mika Yamanaka-Takaichi, et al. This is an open-access article distributed under the terms of the Creative Commons Attribution License, which permits unrestricted use, distribution, and reproduction in any medium, provided the original author and source are credited.

Source of Support: Nil, Conflict of Interest: None declared. 